\title{
INTERACTING BINARIES AND EXTRASOLAR PLANETS: CHALLENGES FOR VLTI
}

\author{
K. D. Gazeas ${ }^{1}$
}

\begin{abstract}
Optical Interferometry can play an important role in modern astronomy. Extrasolar planet and binary star research, are some of the fields, where we will face important discoveries in the future. For the VLTI this will be a real challenge, since it will be the pioneer in angular resolution. In addition, high flux sensitivity will help detecting not only Jupiter-sized planets, but Earth-sized planets too. The ability of the VLTI in detecting new planets, measuring stellar diameters and stellar proper motions, with the use of such techniques, is presented.
\end{abstract}

\section{Introduction}

Using the VLTI, is a unique chance for those who are interested in reaching the limit of observational detectability from ground based observatories. This work of art instrument can offer excellent opportunities for model fitting, since it can provide high flux sensitivity and angular resolution observations and gather interesting information for stellar and planetary research. Mathematical models of binary and multiple stellar systems can determine the stellar parameters and can lead to a study of the stellar formation. With the VLTI we will have the ability to reveal even more information with real observations.

From the study of the interferometric fringes, that we can get with the help of the VLTI, we will have direct measurements of the separation and the brightness ratio of the components in a binary system. In some cases, the diameters of the stars can be found (such those of the Red Pulsating Giants). Astrometrically, the VLTI can determine the orbital motion of the companions, having the potential to find really faint companions in multiple systems, resolving them even inside clusters.

In addition, the excellent quality of data and the high accuracy measurements we can obtain can help in better understanding of the structure of the Active

\footnotetext{
1 Department of Astrophysics, Astronomy and Mechanics, Faculty of Physics, University of Athens, GR 157 84, Zografos, Athens, Greece
} 


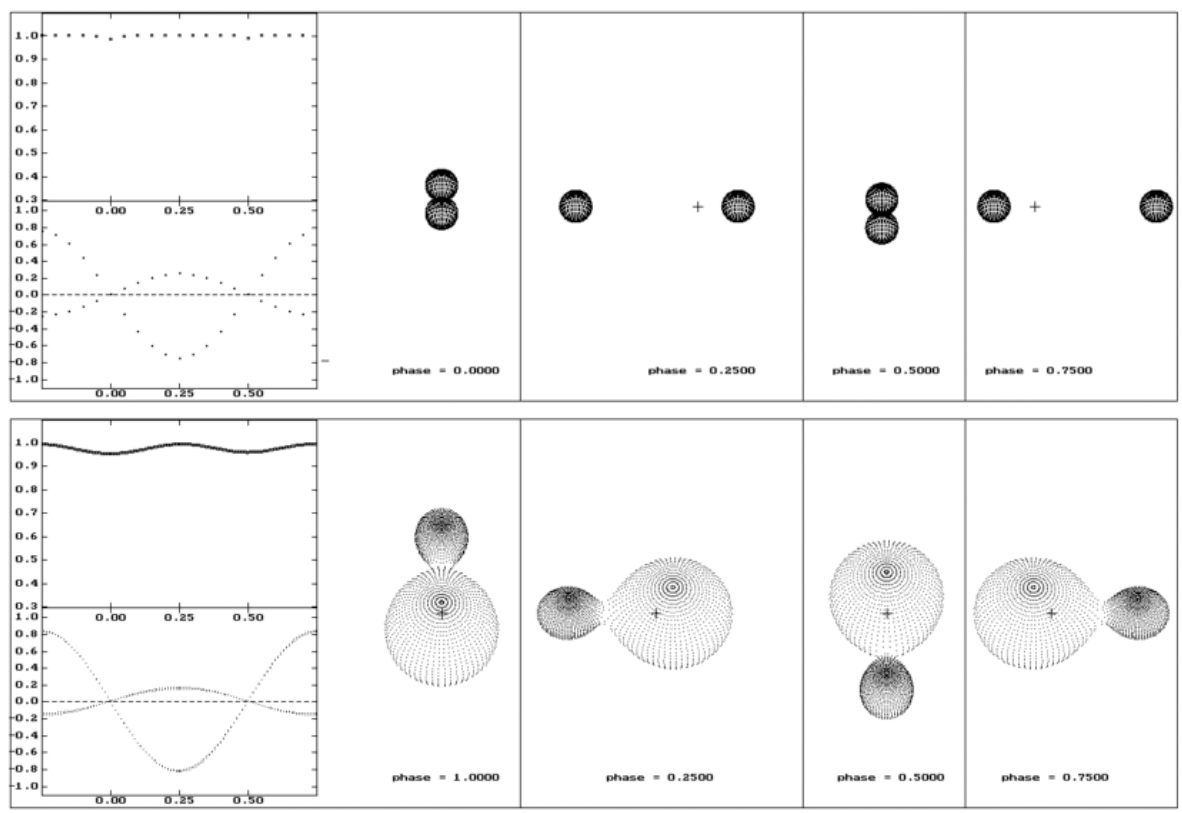

Fig. 1. Simulated lightcurves, radial velocity diagrams and 3D models for partially eclipsing binary systems, detached (above) and contact (below) (produced by Binary Maker $2.0)$.

Galactic Nuclei (AGN) and Quasi Stellar Objects (QSO). The search of companion objects around Young Stellar Objects (YSO), the crowded field astrometry and the stellar structure research are some more of the challenges for the VLTI.

\section{Binary and Multiple Stellar Systems}

On the field of binary stars, there are still some cases that face problems in determining the astrophysical parameters of the system by the use of only photometric and spectroscopic observations. In cases where the luminosity ratio is 10:1 or less, the photometric and spectroscopic measurements are not so confident. The second star is photometrically almost invisible, while in spectroscopic observations is completely absent. The VLTI can reveal information about such systems and determine stellar parameters in partial eclipsing binaries, such as in cases shown in Fig. 1, with extremely low amplitude luminosity variations. Measuring the prime star's gravity motion around the center of the system, we can detect the companion star. Astrometry with the VLTI can be a unique method to measure the star's proper motion.

Moreover, in some cases, the detection cannot only stop to this level. Even 


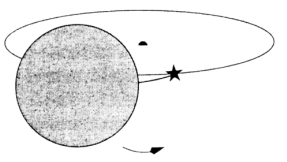

Fig. 2. A theoretical model of the cataclysmic binary Z Cha.

more detailed studies can be done, concerning the fine structure of the system, such as the size of an accretion disk, or the stellar diameters. The cataclysmic binary system Z Cha (Sep. 1, PA 120deg, flux ratio: 10:1) is one of these cases, where the VLTI can detect the flux ratio to separate the contribution between the 2 components and eventually detect the accretion disk (Fig. 2).

Subdwarf B type (sdB) stars such as HS $0705+6700$ can be a challenge for VLTI. They are evolved objects and can be compared with evolved stars in the blue extension of the horizontal branch (HB) but with a very thin hydrogen shell (less than $1 \%$ by mass). The question posed is how the progenitor of a sdB star got rid of most of its hydrogen envelope. Binary evolution with mass transfer and a past common envelope ejection is one scenario, supported by the fact that many sdB stars reside in close binaries (Maxted et al. 2001a,b; Saffer et al. 2001). In such a case, mass transfer should play an important role in the evolution of such binary systems (Drechsel et al. 2001). It is, therefore, crucial to determine the masses of such binaries, especially the eclipsing ones, for a detailed investigation of sdB stars.

\section{Extraxolar Planetary Research}

Finding extrasolar planets is an extremely difficult task. Unlike stars, planets are extremely faint, emitting only thermal infrared radiation. An orbiting planet exerts a gravitational force on its host star, due to the gravitational attraction between the two bodies, resulting the star's position to be perturbed or wobble. This motion uncovers the presence of orbiting planets, even though we cannot see them directly. By measuring this perturbation over several days (or years), it could be calculated as to how big and how far away a planet would have to be to generate such a wobble. The trouble is that this stellar motion appears very small from a great distance. Someone gazing at our sun from 50pc away would see it wobbling in a circle whose radius measures only 1 mas, the limiting resolution for the VLTI.

Using the Doppler technique, over 70 planets have been found so far. Their masses range from about a half to seven times that of Jupiter, their orbital periods span few days to some years, and their distances from their host stars extend from less than one twentieth of Earth's distance to the sun to more than twice that distance. The modern photometric techniques have already discovered or give evidences of such objects. Furthermore, more massive planets or systems closer 


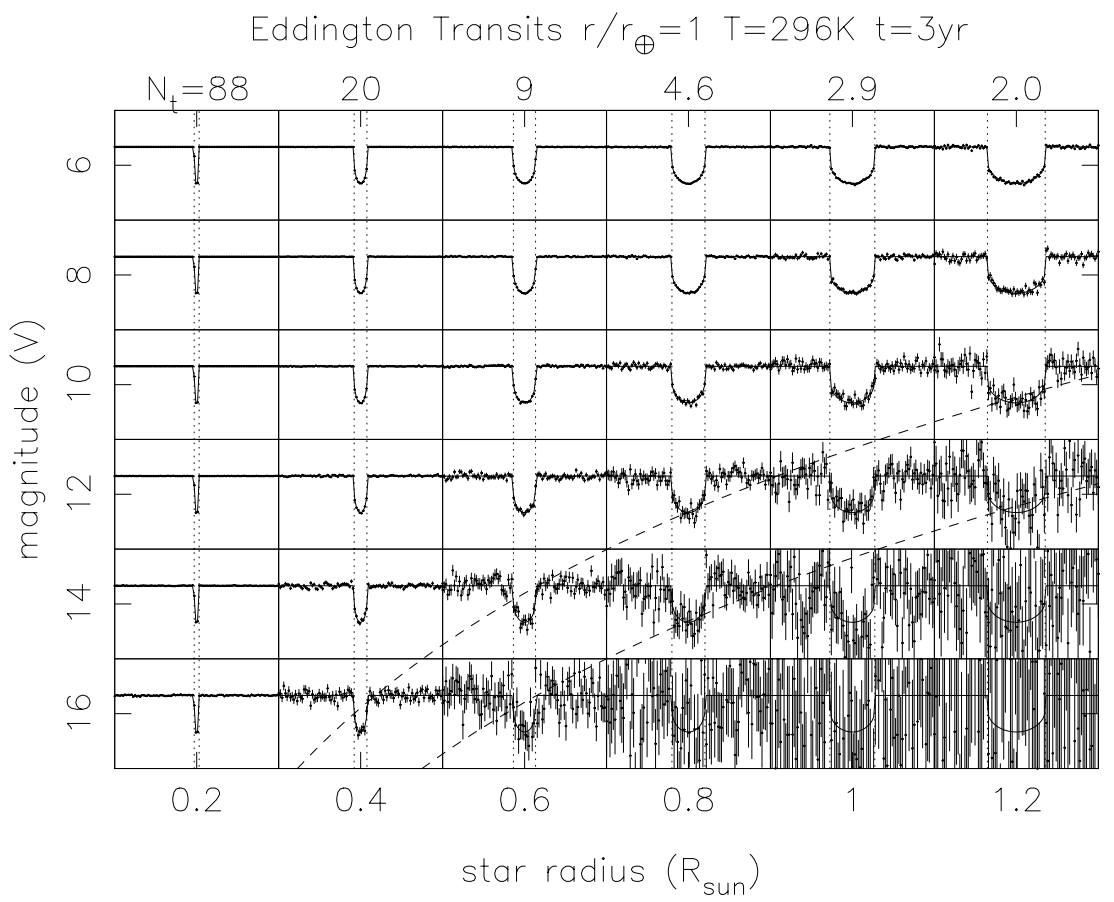

Fig. 3. Simulated lightcurves of Earth-like planets transiting stars with different $V$ magnitude and radius. The simulation is for a planet with the same radius and temperature as Earth, orbiting around its star once every 3 years. The estimate that an Earth-analog system can be detected down to $12-13 \mathrm{mag}$ is confirmed. Such results are expected for the Eddington mission. (after Horne K., 2002).

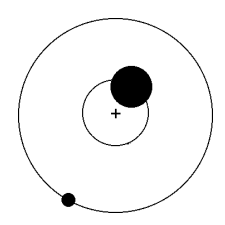

Fig. 4. The star's perturbed orbit around the common mass center of the system.

to us can give even better opportunities for new discoveries. Research projects like the Wide Angle Search for Planets (WASP) are already in action, searching for new worlds, while other are in preparation, such as the Eddington and Kepler missions.

Having the unique angular resolution of 1 mas the VLTI will play an important role in extrasolar planetary research, raising the detectability limit up to $300 \mathrm{pc}$. 


\section{Conclusions}

Photometric and spectroscopic studies are the main observational techniques used today, limited mostly due to the low flux sensitivity. Many of these studies are based on assumptions, since measurements are often uncertain. The use of mathematical models is the only way to check the correctness of the results. Direct measurements are needed in order to make sure that these models are real. Thus, stellar evolution models and stellar population theories will improve.

In addition, numerous extrasolar planets have been found with the modern photometric techniques. In most cases these planets are in systems far away from us. Even though, there are many G-type stars close enough, so that astrometric measurements can reveal a planet from the main star's proper motion. Astrometry in this case can give the results needed for research, unfortunately with the same limitations of sensitivity.

Astrometry with the VLTI is a real pioneer in astronomy. A new era in astronomy will rise in the following years, and new discoveries in various fields are inevitable.

\section{References}

Bradstreet D. H. 1993, BINARY MAKER 2.0 USER MANUAL, Contact Software, Norristown (Pennsylvania)

Drechsel H., Heber U., Napivwotzki R., Ostensen R., Solheim J.E., Johannensen F., Schuh S. L., Deetjen J., Zola S. 2001, A\&A 379, 893

Ferraro F., Paltrinieri B., Fusi Pecci F., et al. 1997, ApJ, 484, L145

Heber U. 1986, A\&A 155, 33

Horne K., 2002, Planetary Transit Surveys, Proceedings from 1st Eddington Workshop on the Stellar Structure and Habitable Planet Finding, Cordoba, 11-15 June 2001, ed. F. Favata, ESA SP-485, p. 137

Kilkenny D., O’Donoghue D., Koen C., Lynas-Gray A. E., van Wyk F. 1998, MNRAS 296,329

Maxted P. F. L., Heber U., Marsh T. R., North R. C. 2001a, MNRAS ???

Maxted P. F. L., Marsh T. R., Heber U. et al. 2001b, MNRAS ???

Menzies J. W., Marang F. 1986, IAUS, 138, 305

Saffer R. A., Bergeron P., Koester D., Liebert J. 1994, ApJ, 432, 351

Saffer R. A., Green E. M., Bowers T. P. 2001, The Binary Origins of Hot Subdwarfs : New Radial Velocities, in Proc. of the 12th European Workshop on White Dwarf Stars, ed. H. P. Shipman, J. L. Provencal, J. McDonald \& S. Goodchild (Astronomical Society of the Pacific: San Francisco), ASP Conf. Ser., 226, 408

Steffen A., Mathieu R.D., Lattanzi M.G., Latham D.W., Mazeh T., Prato L., Simon M., Zinnecker H., Poster Proceedings of IAU Symposium No. 200 on The Formation of Binary Stars, Edited by Bo Reipurth \& Hans Zinnecker, 10-15 April 2000, Potsdam, Germany 